\title{
Patients Carrying 9q31.1-q32 Deletion Share Common Features with Cornelia de Lange Syndrome
}

\author{
Ruixue $\mathrm{CaO}^{\mathrm{a}}$ Tian $\mathrm{Pu}^{\mathrm{a}}$ Shaohai Fang ${ }^{\mathrm{b}}$ Fei Long ${ }^{\mathrm{b}}$ Jing Xie ${ }^{\mathrm{a}}$ Yuejuan $\mathrm{Xu}^{\mathrm{b}}$ \\ Sun Chen ${ }^{\mathrm{a}}$ Kun Sun ${ }^{\mathrm{a}}$ Rang $\mathrm{Xu}^{\mathrm{b}}$ \\ aDepartment of Pediatric Cardiology, Xinhua Hospital, Shanghai Jiao Tong University School of \\ Medicine, bScientific Research Center, Xinhua Hospital, Shanghai Jiao Tong University School of \\ Medicine, Shanghai, PR. China
}

\section{Key Words}

Cornelia de Lange Syndrome • 9q31.1-q32 deletion • SMC2 • Exome sequencing

\begin{abstract}
Background: Cornelia de Lange Syndrome (CdLS) is a rare but severe clinically heterogeneous developmental disorder characterized by facial dysmorphia, growth and cognitive retardation, and abnormalities of limb development. Objectives: To determine the pathogenesis of a patient with CdLS. Methods: We studied a patient with CdLS by whole exome sequencing, karyotyping and Agilent CGH Array. The results were confirmed by quantitative real-time PCR analysis of the patient and her parents. Further comparison of our patient and cases with partially overlapping deletions retrieved from the literature and databases was undertaken. Results: Whole exome sequencing had excluded the mutation of cohesion genes such as NIPBL, SMC1A and SMC3. The result of karyotyping showed a deletion of chromosome 9q31.1-q32 and the result of Agilent CGH Array further displayed a 12.01-Mb region of deletion at chromosome bands 9q31.1-q32. Reported cases with the deletion of 9q31.1-q32 share similar features with our CdLS patient. One of the genes in the deleted region, SMC2, belongs to the Structural Maintenance of Chromosomes (SMC) family and regulates gene expression and DNA repair. Conclusions: Patients carrying the deletion of 9q31.1-q32 showed similar phenotypes with CdLS.
\end{abstract}




\section{Introduction}

Cornelia de Lange Syndrome (CdLS), also known as Brachman de Lange syndrome, is a rare but severe clinically heterogeneous developmental disorder characterized by distinctive facial features, growth and cognitive retardation, and abnormalities of limb development [1]. The facial features are the most clinically consistent and recognizable characteristics of CdLS, including underdeveloped orbital arches, long curly eyelashes, thin lips, downturned angle of the mouth, hirsutism, short neck with low anterior and posterior hairlines, anteverted nares, well defined and arched (pencil-like) eyebrows, and long philtrum [2]. The incidence of CdLS varies from $1 / 10,000$ to $1 / 60,000$ of live births. However, due to possibly misdiagnosed cases, the exact incidence is still unclear [3].

Approximately $60 \%$ of CdLS patients carried mutations in NIPBL, $5 \%$ of CdLS patients had mutations in $S M C 1 A$, and one individual was found to have a deletion in SMC3. The haploinsufficient mutations of NIPBL (such as nonsense mutations, splice site mutations, and out-of-frame deletions or insertions) usually result in a more severe growth and cognitive retardation, as well as abnormalities of limb development. However, patients with mutations in SMC1A and SMC3 only have mild or moderate phenotypes without distinct impairments or structural abnormalities of the limb or other organ systems [4]. Most genes associated with CdLS patients, including NIPBL, SMC1A and SMC3, are cohesin related genes. Studies of other genes, such as MRPS22 and HDAC8, and an 8p23.1 deletion are ongoing [5-7].

To date, approximately $35 \%$ of clinically diagnosed CdLS patients do not carry any identifiable mutation, which indicates that some disease-related genes remain to be discovered. In this study, we analyzed via whole exome sequencing, karyotyping and aCGH the genetics of an 18-month-old female CdLS patient with facial dysmorphism, mental retardation, developmental delay, a small atrial septal defect (ASD), hearing disorder, and irregular dentition. After excluding the mutations of these cohesion genes by whole exome sequencing, we detected a $12.01 \mathrm{Mb}$ deletion in 9q31.1-q32 which may be associated with CdLS. After a comparison of our case with 11 previously reported cases with partially overlapping deletions, we found that all of these cases shared many features with CdLS, which indicated that they might have a common pathogenesis that caused these features. Thus we suggest that some genes in 9q31.1-q32 may be associated with these common features and may be candidate genes in the cohort of CdLS patients in whose DNA no mutation of known pathogenic genes has yet been discovered.

\section{Materials and Methods}

\section{Whole Exome Sequencing}

Genomic DNA was fragmented and hybridized to the capture array according to the manufacturer's protocol of QIAamp DNA Blood Midi Kit, and the enriched DNA fragments were eluted and amplified with ligation-mediated PCR through the linkers added to the exonic DNA fragments. Before the second run of library construction, qPCR was performed to estimate the degree of enrichment of the exonic sequences. A minimum requirement of an 80 -fold enrichment was achieved for all of the libraries prepared for the next procedure. The enriched exonic DNA fragments were randomly blunt-end ligated with DNA ligase to fragments ranging in size from $2-5 \mathrm{~kb}$. The resultant DNA products were sheared to $200 \mathrm{bp}$ on average and were subjected to standard Illumina hiseq2500 library preparation according to the Illumina protocol.

Exome-enriched shotgun libraries were sequenced on the Illumina HiSeq 2500 platform, and paired-end reads with an average size of $100 \mathrm{bp}$ were generated. Image analysis and base calling were performed with the Illumina's Consensus Assessment of Sequence and Variation (CASAVA) 1.8, using default parameters. After removing reads with sequence matching the sequencing adaptors and low-quality reads with more than five unknown bases, high-quality reads were aligned to the NCBI human reference genome (hg19) using Burrows-Wheeler Aligner (BWA) version 0.6.2 [8] with the default parameters. To identify potential mutations, we performed local realignments of the BWA reads using the Genome Analysis Toolkit (GATK) [9]. Mutations including nonsynonymous mutations, stopgain mutations, stoploss mutations, splice 
Cao et al.: 9q31.1-q32 Deletion Patients and CdLS Have Common Features

Table 1. The primers of qPCR

\begin{tabular}{lll}
\hline Gene & Primer-F $\left(5^{\prime}-3^{\prime}\right)$ & Primer-R $\left(5^{\prime}-3^{\prime}\right)$ \\
\hline SMC2 & ATTTTATTTTAGGTTCGGGCTT & CAATCCTCTTAGTGATAGAATGTGC \\
CANNAL2 & ACTGTGCATCTTCATATTGCC & AGTTTCTCACCTCGTCTTCCTT \\
DFNB31 & CCTGTTCCACCTTCTAGTAG & GATTCGAACTCAGGCTGGTC \\
\hline
\end{tabular}

site mutations, frameshift deletions or insertions, and noneframeshift deletions or insertions in the coding sequences of genes would be identified as functional mutations.

\section{Karyotyping}

The karyotypes were examined by G-banding at the level of 550. The peripheral blood samples were collected with heparin added as an anticoagulant from the patient and parents and cultured in RPMI1640 culture medium with $20 \%$ calf serum (Invitrogen Gibco, USA) at $37^{\circ} \mathrm{C}$ in $5 \%$ CO2. Preparation of metaphase and conventional cytogenetics followed standard laboratory procedures. At least 20 banded metaphases with good chromosome separation were analyzed by experienced geneticists. Commercially available locusspecific probe kits N25 (D22S75) and TUPLE1 (HIRA) were purchased from Vysis (Downers Grove, IL, USA).

\section{Agilent CGH Array $4 \times 180 \mathrm{~K}$}

Peripheral blood samples were exsanguinated into an EDTA anticoagulant tube. DNA was extracted with the QIAamp DNA Blood Midi Kit (Qiagen, Duesseldorf, Germany) following the manufacturer's instructions. Purified genomic DNA was resuspended in ddH2O for SNP-array analysis or in Tris-EDTA for other experiments, and DNA stocks were stored at $-80^{\circ} \mathrm{C}$. Whole-genome aCGH was performed on the patient's blood using an Agilent CGH Array $4 \times 180 \mathrm{~K}$, which has 170,334 probes and a median resolution of $13 \mathrm{~kb}$ across the entire genome according to the manufacturer's instruction. The sample passed initial QC and was submitted for copy number various (CNV) discovery. High-confidence CNVs were detected by ADM-2 with the minimum marker 5 and threshold 6.0. The data was filtered, and only those regions larger than $100 \mathrm{~kb}$ comprising at least 50 contiguous markers were retained. The details of this experiment were deposited in dbVar (http://www.ncbi.nlm.nih.gov/dbvar) and the accession number is GSE54354 (NCBI GEO). Finally, the results were compared to known CNVs in the Database of Genomic Variants (DGV, http:// dgvbeta.tcag.ca/dgv/app/home) and Online Mendelian Inheritance in Man (OMIM, http://www.ncbi.nlm. nih.gov/omim/) to distinguish common CNVs from CNVs likely to be causal.

\section{qPCR Validation}

For each tested CNV, at least three primer pairs in three genes were designed within the boundaries of the CNV region (Table 1). Small portions of genomic DNA extracted from healthy people were mixed to form a DNA pool serving as the normal control, while the gene ALB, which has few variations, was used as the control gene in qPCR. These genes were quantified to determine the copy number (CN), as were pivotal genes nearby. When results differed between aCGH and qPCR, the qPCR results were used. When the results were consistent, the segments were further validated in the patient's parents.

\section{Results}

\section{Clinical description}

The child was diagnosed with CdLS when she was eighteen months old. Now, she is two years old. She is the only child in the family, and there is no family history of congenital malformations. Her mother was 18 years old and her father was 20 years old at her birth, and her parents are non-consanguineous. At 41 weeks of gestation, she was born with a dyspnea and distinctive facial features (a large protruding left ear, underdeveloped orbital arches, long curly eyelashes, thin lips, downturned angle of the mouth, short neck with low anterior and posterior hairlines, anteverted nares, well defined and arched eyebrows, long philtrum, anterior median fissure and micrognathia) (see Fig. 1). She was born by a cesarean section delivery due to oligohydramnios and lived in the newborn ICU for 15 days. Her birth 
Fig. 1. Our CdLS patient with distinctive facial features. A large protruding ear, underdeveloped orbital arches, long curly eyelashes, thin lips, downturned angle of the mouth, short neck with low anterior and posterior hairlines, anteverted nares, well-defined and arched eyebrows, long philtrum, anterior median fissure, and micrognathia.

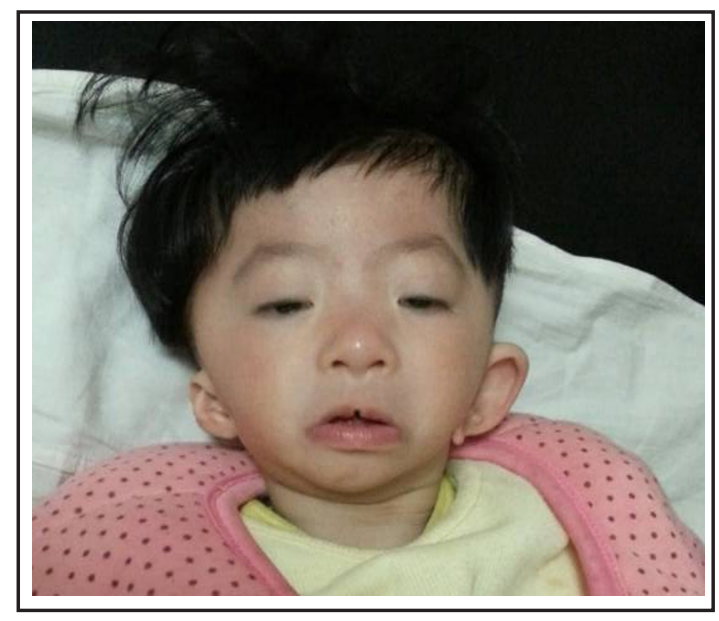

Fig. 2. Echocardiography images of this case. +: the endpoints of the ASD.

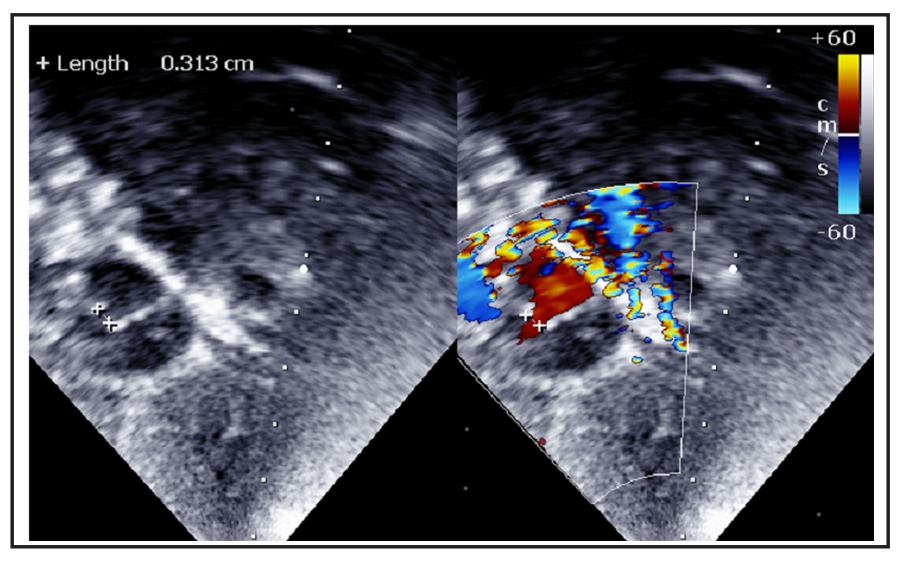

Fig. 3. The karyotype of 46,XX,del(9) (q31.1q32). The arrow at chromosome 9 indicates the breakpoint.

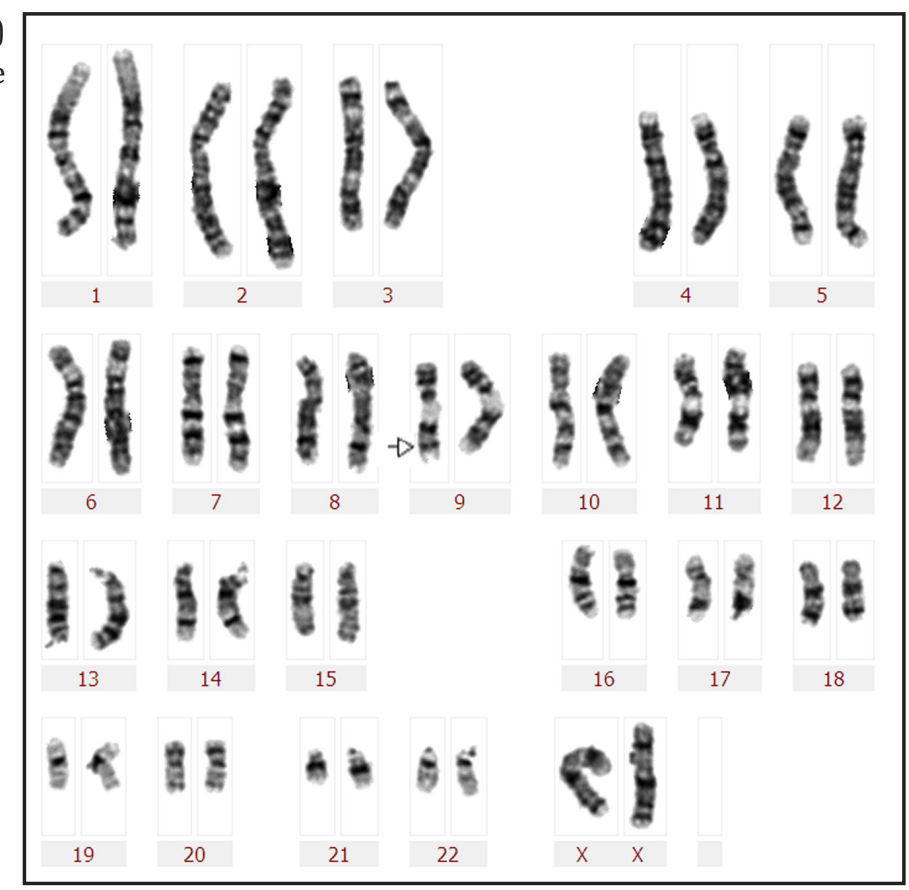

weight was 1300g (a very low birth weight for infants), and her birth length was $48 \mathrm{~cm}$. At the age of 1 month, she was diagnosed with ASD (see Fig. 2). Then, she grew up with obvious development delay. She could raise her head when she was 5 months old, sit at 10 months and now she still can't walk or speak. She is always aggressive and hard to communicate 


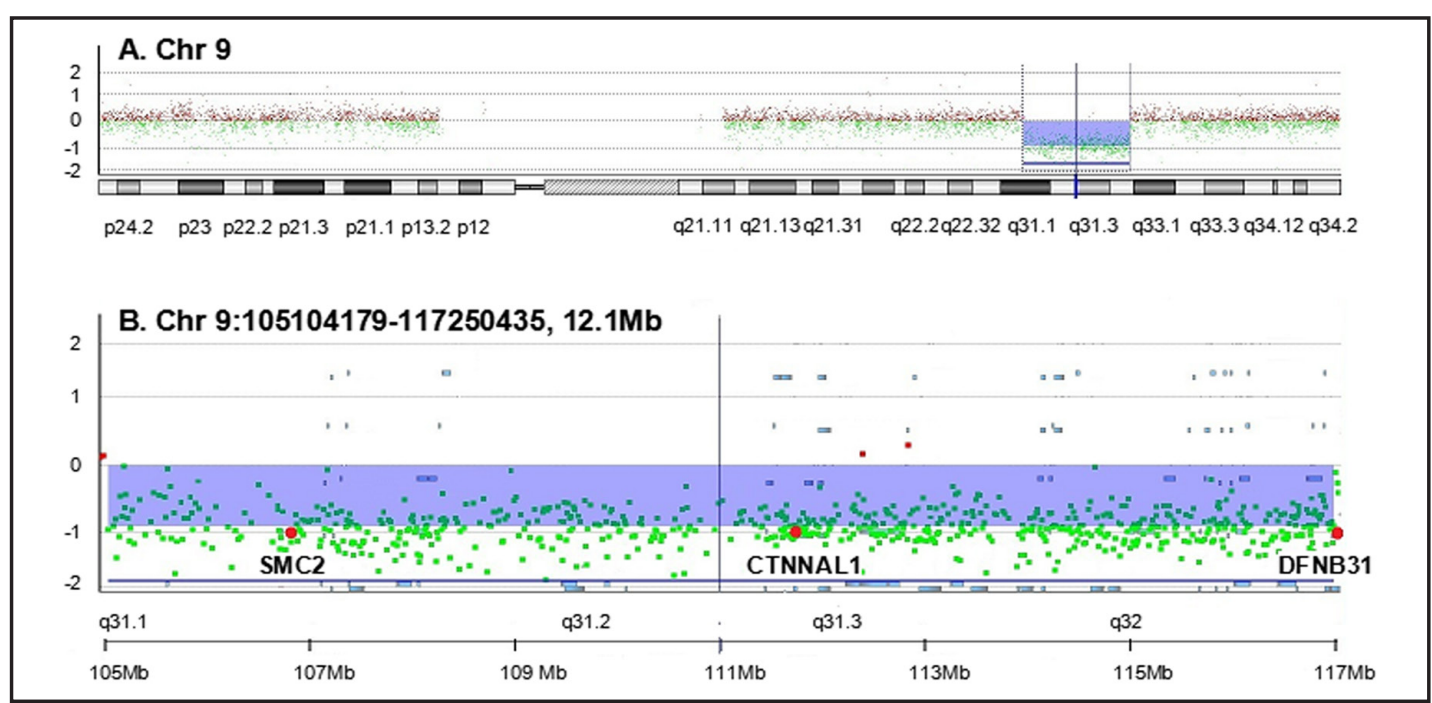

Fig. 4. Results of aCGH on peripheral blood. (A) Chromosomal view of the 12.1-Mb deletion at chromosome bands 9q31.1-q32[arr 9q31.1q32 (105,104,179-117,250,435) × 1] (NCBI build 37) and (B) zoomed-in view. Red spots indicate the three genes we verified by qPCR.

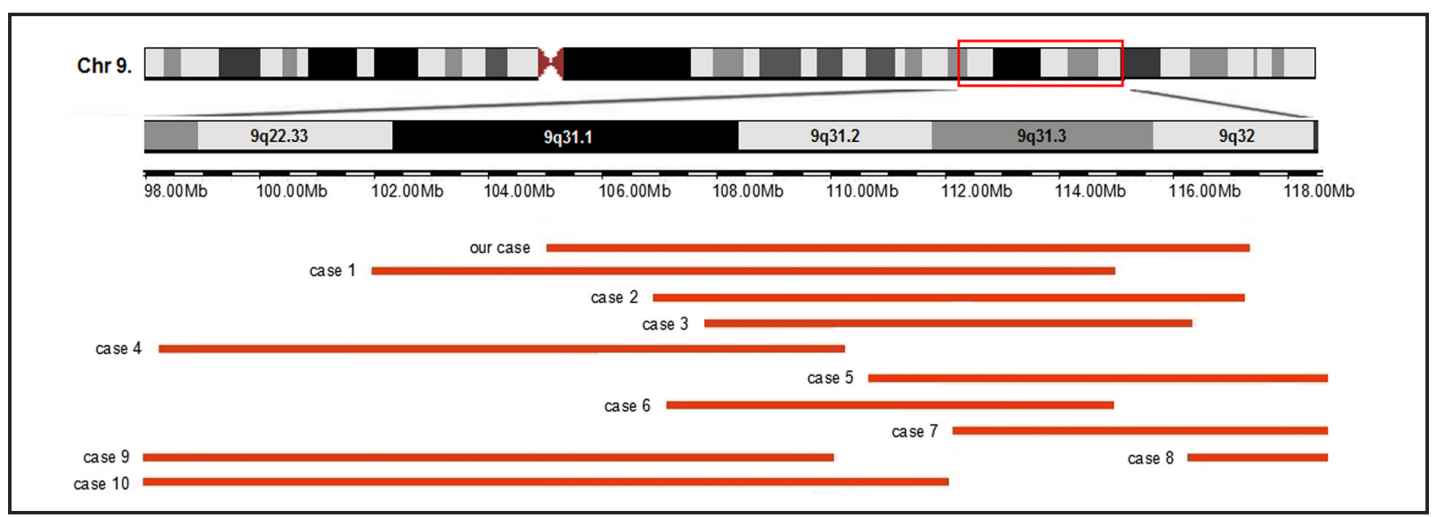

Fig. 5. Schematic representation of our case and the 11 previously presented cases having $9 q$ deletions partially overlapping with the region of chr:9q31.1-32(105190105-117195154). The physical positions of the base pair here are based on GRch/hg19. Case 1 (DECIPHER ID: 250887). chr 9: q22.32 - 9q32(101997966115022084). hg19. Case 2[11]. chr9:106,859,697-117,190,101. hg19. Case 3[10]. chr9q31.1-q31.3 (107970kb-114341kb). hg19. Case 4 (DECIPHER ID: 270439). chr9:q22.32 - 9q31.2(98218538-110294149). hg19. Case 5[12]. chr9q31.1-q33.1 (110672051-120997502). hg19. The base pairs of case 6[13], case 7[14] and case 8[14] were estimated according to $106.2-113.27 \mathrm{Mb}, 111,330,304-121,013,839$, and 115,369,807121,843,479 based on NCBI36/hg18. Case 9[15] and Case 10[16]. chr9q22.3-q31.3 (94Mb-106Mb) based on NCBI35/hg17 and chr9q22.32-9q33.2, the base position was unclear.

with. At the age of 17 months, she was diagnosed with hearing disorder. At the age of 18 months, her weight was $8000 \mathrm{~g}$ ( $<$ middle weight- 2 SD), and her height was $73 \mathrm{~cm}(<$ average height -3 SD). According to the Gesell Developmental Scale, at the age of 18-months, her age equivalent in months is baby at 11-month developmental level. Furthermore, she has irregular dentition and dyspepsia and is diagnosed with CdLS.

\section{Molecular and cytogenetic data}

Whole exome sequencing of the CdLS patient revealed no functional mutations in the coding sequences of genes associated with CdLS such as NIPBL, SMC1A, SMC3, MRPS22 and HDAC8. Karyotyping analysis of the patient's peripheral blood showed that the child had a deletion at chromosome bands 9q (see Fig. 3) while no obvious structural or numerical 
Table 2. The characteristics phenotypes of the previous patients with an overlapping deletion with 9q31.1q32 compared with the diagnostic criteria for CdLS. 1. Eyebrow: synophrys or well defined and arched eyebrows. 2. Ears: dysplastic helices, antihelices or low-set ears. 3. Nose: broad and/or depressed nasal bridge, anteverted nares. 4. Mouth: thin lips, downturned angle of the mouth. 5. (1) Hand anomalies: tapered fingers, camptodactyly, small hands and short digits, proximally placed thumbs, and/or small/hypoplastic nails; (2) Foot anomalies: syndactyly of toes, and/or small feet and short toes; 6. Gastrointestinal/respiratory systems: Gastroesophageal reflux; twisted bowels; gut duplication; oesophageal stenosis;pyloric stenosis; congenital diaphragmatic hernia. 7. Hair development : Excessive body and facial hair; long eyelashes. 8. NS: Neoplasm of the skin ; G: Gibbus; S: Scoliosis; H: Hypertelorism

\begin{tabular}{|c|c|c|c|c|c|c|c|c|c|c|c|c|}
\hline Features of the CdLS phenotype & Our case & 1 & 2 & 3.1 & 3.2 & 4 & 5 & 6 & 7 & 8 & 9 & 10 \\
\hline Growth retardation & + & + & + & + & + & + & + & + & + & + & + & + \\
\hline \multicolumn{13}{|l|}{ Neurodevelopment } \\
\hline Psychomotor development & + & + & + & + & + & + & + & + & + & + & + & + \\
\hline Communicate skills & + & + & + & - & + & + & + & + & + & + & + & + \\
\hline Autism & - & - & + & - & - & - & - & + & - & - & - & - \\
\hline Seizures & - & - & - & - & - & - & - & - & - & + & + & - \\
\hline Self-injurious behaviour & - & - & - & - & - & - & - & - & - & - & - & - \\
\hline \multicolumn{13}{|l|}{ Craniofacial development } \\
\hline Microcephaly & - & - & + & - & - & - & - & + & - & + & - & - \\
\hline Eyebrow ${ }^{1}$ & + & - & - & + & + & + & + & - & - & + & + & + \\
\hline Ears $^{2}$ & + & + & - & - & + & - & + & + & + & - & - & + \\
\hline Nose $^{3}$ & + & + & - & + & - & + & + & + & + & + & + & + \\
\hline Mouth 4 & + & - & - & - & - & - & - & - & + & - & - & - \\
\hline Small Jaw & + & - & - & - & - & - & - & - & + & + & - & + \\
\hline High-arched Palate & + & + & - & - & - & + & - & - & + & - & + & + \\
\hline Clefting & + & + & - & - & - & - & - & - & - & - & - & + \\
\hline Short Neck & + & - & - & + & - & + & + & + & + & - & - & + \\
\hline \multicolumn{13}{|l|}{ Limb abnormalities 5} \\
\hline Foot anomalies & - & - & - & - & - & - & + & + & - & + & + & - \\
\hline Hand anomalies & + & + & + & + & - & - & + & + & - & + & - & + \\
\hline Gastrointestinal/respiratory systems 6 & $6+$ & - & + & - & + & + & - & - & - & - & - & + \\
\hline Hearing Loss & + & + & + & - & - & + & + & - & - & - & - & - \\
\hline Kidney development & - & + & - & - & - & - & - & + & - & - & - & + \\
\hline Cardiac problems & + & + & + & + & + & - & + & - & - & - & + & - \\
\hline Hypoplastic male genitalia & - & - & - & - & - & + & + & - & - & - & + & - \\
\hline Hair development ${ }^{7}$ & + & - & - & + & + & - & - & - & + & - & - & + \\
\hline Dental problems & + & - & - & - & - & + & + & + & - & - & - & + \\
\hline Others $^{8}$ & - & - & $\mathrm{H}$ & G & G & NS & $\mathrm{S}$ & - & $\mathrm{S}$ & - & $\mathrm{H}$ & NS \\
\hline
\end{tabular}

abnormalities were found in the metaphase spreads of her parents. Further analysis of aCGH showed that she carried a 12.01-Mb deletion region at chromosome bands 9q31.1-q32 $(105,190,105-117,195,154)$, which is a single gene allele loss (see Fig. 4), and the deletion of 8p23.1 was not discovered. The deleted region encompasses 22 OMIM genes including SMC2, a member of Structural Maintenance of Chromosomes (SMC) family. Then the deletion of 9q31.1-q32 was confirmed in the child by qPCR, while her parents did not have this deletion.

\section{Discussion}

Our patient is an 18-month-old female CdLS patient with facial dysmorphism, mental retardation, developmental delay, a small ASD, hearing disorder, and irregular dentition. In order to study the probable pathogenesis of this CdLS patient, we first studied the CdLS patient by whole exome sequencing. However, there was no mutation of previously-shown related genes such as NIPBL, SMC1A, SMC3, MRPS22 and HDAC8. Thus, there still must be 
some undiscovered pathogenesis. Using karyotyping and Agilent CGH Array analysis, we detected a 12.01-Mb deletion encompassing 22 OMIM genes at chromosome band 9q31.1-q32(105190105-117195154).

\section{Patients with deletion of 9q31.1-q32 showed similar features with CdLS}

CdLS is characterized by distinctive facial features, growth and cognitive retardation, and abnormalities of limb development. While growth and cognitive retardation are common features of many syndromes, the distinctive facial features and limb development abnormalities play key roles in the diagnosis. Research in the USA showed that an average of $75 \%$ of cases was correctly diagnosed by only facial photographs. The emphasis was placed on eyebrows and nasal features. Upper lip and micrognathia were also important characteristics[2].

Karyotyping and Agilent CGH Array analysis showed that the patient with CdLS carried a deletion of 9q31.1-q32. However, reports about the deletion of chromosome $9 q$ are rare and only about 20 patients have been described in medical literature, spanning the breakpoints from 9q21 to 9q34 [10]. To date, no well-defined syndrome has been specifically associated with this region. A comparison of our patient with the 11 previously reported cases with partially overlapping deletions is provided in Table 2 and their deletion regions are compared in Fig. 5 (case 1 and case 4 came from DECIPHER, http://decipher.sanger.ac.uk/) [10-16].

It's interesting to note that the phenotypes of most patients carrying a deleted region overlapping with our proband have similar features including postnatal growth retardation, intellectual disability, craniofacial anomalies, and limb abnormalities (see Table 2). Nearly all phenotypes are those of CdLS, except neoplasm of the skin, which is rare in CdLS. Both case 4 and case 10 have this phenotype. After reviewing genes in the deleted region of case 4, case 10, we found a gene PTCH1 (located in chr9:98247867-98279247). Mutations of PTCH1 have been identified to be responsible for nevoid basal cell nevus syndrome (NBCCS) in many cases [17-19]. Both haploinsufficiency and duplication of PTCH1 could cause NBCCS [20,21]. Therefore, we deduced that maybe PTCH1, which is not in our deletion region, was the cause of neoplasm of the skin. Though it's inadequate to say that the 11 cases are completely in accordance with the diagnostic criteria of CdLS, it's true that they share similar phenotypes with CdLS.

Candidate genes for these common phenotypes or CdLS in 9q31.1-q32

To date, no well-defined syndrome has been specifically associated with 9q31.1-q32 and no part of the deletion region is shared by all the cases. It's very difficult to correlate these phenotypes with specific genes in such a large microdeletion. Fortunately, these phenotypes are similar to those of CdLS. Maybe some genes that have not been reported are associated with CdLS or these common features. Keeping this in mind, we first analyzed the pathogenesis that had been widely accepted in CdLS and tried to find some candidate genes.

SMC2. SMC1A, SMC2 and SMC3 are members of the SMC family. The SMC proteins share highly similar domain structures. SMC2 and SMC4 constitute the condensin complexes, which have structures exactly like that of the SMC1/3 cohesin complex: a V-shaped structure with two long coiled-coil arms, each containing an ATP-binding cassette (ABC) head domain at the distal end [22]. Mutations in SMC1A and SMC3 had been identified in CdLS patients with mild or moderate phenotypes. SMC1A and SMC3 are essential for regulating gene expression, DNA repair and sister chromatid cohesion [23]. In mammals, cohesin including SMC1A and SMC3 accumulate at sites where CCCTC-binding factor (CTCF) is localized [24]. Knockdown of cohesin alone resulted in deregulated gene expression among CTCF targets [25]. These studies indicated that the phenotypes of CdLS may be due to defects in the function of cohesin and CTCF in regulating gene activities [26-29].

The functions of SMC2 and SMC1A, SMC3 are highly similar and related. SMC2 play key roles in DNA repair and correct progression during mitosis. Condensin could act against replication protein $A$ in mitosis and interphase by promoting DNA annealing [30]. The mutation in the hinge domain of $S M C 2$ resulted in severely reduced viability during mitosis 
after exposure to DNA damaging agents at permissive temperatures. Besides, SMC2 is required for the correct progression of sister chromatid cohesion [31].

Previous studies confirmed the role of SMC2 in gene regulation [32, 33]. More recently, it has been discovered that $S M C 2$ can negatively regulate rRNA gene transcription via CTCF, which is colocalized with and required for cohesin. SMC2 and CTCF bind to a specific rDNA locus in a competitive manner. SMC2 knockdown promotes the loading of CTCF onto the rDNA locus and facilitates CTCF-enhanced rRNA gene transcription [34]. While the severe phenotypes of CdLS are related with the defect in cohesin function of regulating gene expression by CTCF, SMC2 can regulate gene transcription by CTCF. These findings indicate that SMC2 may cause CdLS via the defect in regulating CTCF-associated gene expression and DNA repair.

DFNB31. There is no relationship between DFNB31 and function of gene regulation or DNA repair. DFNB31, which is located in 9q31.2, is a gene responsible for autosomal recessive non-syndromic hearing loss (ARNSHL) [35]. ARNSHL is the most common and severe form of hereditary hearing impairment, accounting for approximately $80 \%$ of cases of genetic deafness. However, in CdLS patients, hearing loss is perceived as an atypical feature. About $40-80 \%$ of cases may carry this phenotype [2]. Our patient was diagnosed with hearing disorder at the age of 17 months. Thus, it's a question whether deafness is a part of CdLS or another disease apart from CdLS. As ARNSHL is an autosomal recessive genetic disease and there is one chromosome microdeletion in our patient, so if the hearing disorder were caused by ARNSHL, there must be a mutation of the DFNB31 in the other chromosome [36]. Keeping this in mind, we searched for the mutations of DFNB31 in our patient. However, the result of whole exome sequencing showed that there was no functional mutation of DFNB31 in this patient. Consistent with the incidence of hearing loss in CdLS, in all 12 patients we have described above, there are five $(41.7 \%)$ patients suffering from deafness. Among these five patients, only two cases' deletion region contained the gene DFNB31. Thus, maybe the hearing disorder of these patients was a part of CdLS, but not due to DFNB31.

Other genes. SMC2 haploinsufficiency may play a role in the occurrence of some clinical features in this patient. Only 6 cases have the deletion of SMC2 (Fig. 5) and there is no specific phenotype in patients with SMC2. Thus, there still must be some other genes that contribute to these phenotypes. The functions of genes in chr9:98247867-98279247 need to be further studied.

\section{Conclusion}

In our study, we first excluded the mutations of CdLS related genes which had been reported previously such as NIPBL, SMC1A, SMC3, MRPS22 and HDAC8. Then, we detected a 12.01-Mb deletion at chromosome band 9q31.1-q32 by karyotyping and aCGH array. The comparison of our patient with 11 previously reported cases with partially overlapping deletions showed similar phenotypes and almost all phenotypes are similar to those of CdLS. Gene SMC2 may play key roles in CdLS or these common phenotypes by influencing the function of gene expression and DNA repair. However, with access to only one patient, we cannot verify the role of the 12.01-Mb deletion at chromosome band 9q31.1-q32 and the function of SMC2 among a larger population of CdLS patients, so further studies should be performed.

\section{Ethics Statement}

Ethics committee of Xinhua Hospital specifically approved of this study and written informed consents were obtained from her parents. The parents of the individual in this manuscript have given written informed consent to publishing these case details. 
Cao et al.: 9q31.1-q32 Deletion Patients and CdLS Have Common Features

\section{Abbreviations}

Cornelia de Lange Syndrome (CdLS);, atrial septal defect (ASD); Consensus Assessment of Sequence and Variation (CASAVA); Burrows-Wheeler Aligner (BWA); Genome Analysis Toolkit (GATK); copy number various (CNV); copy number (CN); nevoid basal cell nevus syndrome (NBCCS); structural maintenance of chromosomes (SMC); ATP-binding cassette (ABC); CCCTC-binding factor (CTCF); autosomal recessive non-syndromic hearing loss (ARNSHL).

\section{Acknowledgments}

We thank the family for their generous participation. The project was funded by the grant (2010CB529500) from the National Basic Research Program of China, the grants (81070135/H0204, 81300068/H0201) from the National Natural Science Foundation of China, the grant from Shanghai university top discipline B constructions program, the grant of the Science Committee of Shanghai (13JC1401705). The funders had no role in study design, data collection and analysis, decision to publish, or preparation of the manuscript. The authors would like to acknowledge Yifan Zhu for language editing.

\section{Disclosure Statement}

The authors declare that they have no competing interests.

\section{Reference}

1 Dorsett D, Krantz ID: On the Molecular Etiology of Cornelia de Lange Syndrome. Ann N Y Acad Sci 2009;1151:22-37.

2 Mikolajewska E: Interdisciplinary therapy in Cornelia de Lange syndrome - review of the literature. Adv Clin Exp Med 2013;22:571-577.

3 Barisic I, Tokic V, Loane M, Bianchi F, Calzolari E, Garne E, Wellesley D, Dolk H: Descriptive epidemiology of Cornelia de Lange syndrome in Europe. Am J Med Genet A 2008;46A:51-59.

-4 Deardorff MA, Kaur M, Yaeger D, Rampuria A, Korolev S, Pie J, Gil-Rodríguez C, Arnedo M, Loeys B, Kline AD, Wilson M, Lillquist K, Siu V, Ramos FJ, Musio A, Jackson LS, Dorsett D, Krantz ID: Mutations in Cohesin Complex Members SMC3 and SMC1A Cause a Mild Variant of Cornelia de Lange Syndrome with Predominant Mental Retardation. Am J Hum Genet 2007;80:485-494.

-5 Ratajska M, Wierzba J, Pehlivan D, Xia Z, Brundage EK, Cheung SW, Stankiewicz P, Lupski JR, Limon J: Cornelia de Lange syndrome case due to genomic rearrangements including NIPBL. Eur J Med Genet 2010;53:378-382.

-6 Chen CP, Lin SP, Liu YP, Chern SR, Wu PS, Su JW, Chen YT, Lee CC, Wang W: An interstitial deletion of 8q23.3-q24.22 associated with Langer-Giedion syndrome, Cornelia de Lange syndrome and epilepsy. Gene 2013;529:176-180.

7 Deardorff MA, Bando M, Nakato R, Watrin E, Itoh T, Minamino M, Saitoh K, Komata M, Katou Y, Clark D, Cole KE, De Baere E, Decroos C, Di Donato N, Ernst S, Francey LJ, Gyftodimou Y, Hirashima K, Hullings M, Ishikawa Y, Jaulin C, Kaur M, Kiyono T, Lombardi PM, Magnaghi-Jaulin L, Mortier GR, Nozaki N, Petersen MB, Seimiya H, Siu VM, Suzuki Y, Takagaki K, Wilde JJ, Willems PJ, Prigent C, Gillessen-Kaesbach G, Christianson DW, Kaiser FJ, Jackson LG, Hirota T, Krantz ID, Shirahige K: HDAC8 mutations in Cornelia de Lange syndrome affect the cohesin acetylation cycle. Nature 2012;489:313-317.

-8 Liu Y, Schmidt B, Maskell DL: CUSHAW: a CUDA compatible short read aligner to large genomes based on the Burrows-Wheeler transform. Bioinformatics 2012;28:1830-1837. 
Cao et al.: 9q31.1-q32 Deletion Patients and CdLS Have Common Features

-9 McKenna A, Hanna M, Banks E, Sivachenko A, Cibulskis K, Kernytsky A, Garimella K, Altshuler D, Gabriel S, Daly M, DePristo MA: The Genome Analysis Toolkit: a MapReduce framework for analyzing nextgeneration DNA sequencing data. Genome Res 2010;20:1297-1303.

10 Mucciolo M, Magini P, Marozza A, Mongelli P, Mencarelli MA, Hayek G, Tavalazzi F, Mari F, Seri M, Renieri A, Graziano C: 9q31.1q31.3 deletion in two patients with similar clinical features: a newly recognized microdeletion syndrome? Am J Med Genet A 2014;164A:685-690.

-11 Chien SC, Li YC, Li LH, Wu JY, Hsu PC, Shi SL, Tsai FJ, Lin CC: A new familial insertion, ins(18;9) (q12.2;q33.1q31.1) with a 9q31.1-9q33.1 deletion in a girl with a cleft lip and palate. Am J Med Genet A 2010;152A:1862-1867.

-12 Xu M, Zhou H, Yong J, Cong P, Li C, Yu Y, Qi M: A Chinese patient with KBG syndrome and a 9q31.2-33.1 microdeletion. Eur J Med Genet 2013;56:245-250.

13 Gamerdinger U, Eggermann T, Schubert R, Schwanitz G, Kreiss-Nachtsheim M: Rare interstitial deletion 9q31.2 to q33.1 de novo: longitudinal study in a patient over a period of more than 20 years. Am J Med Genet A 2008;146A:1180-1184.

14 Kulharya AS, Flannery DB, Norris K, Lovell C, Levy B, Velagaleti GV: Fine mapping of breakpoints in two unrelated patients with rare overlapping interstitial deletions of $9 \mathrm{q}$ with mild dysmorphic features. Am J Med Genet A 2008;146A:2234-2241.

15 Chen CP, Lin SP, Wang TH, Chen YJ, Chen M, Wang W: Perinatal findings and molecular cytogenetic analyses of de novo interstitial deletion of 9q (9q22.3-->q31.3) associated with Gorlin syndrome. Prenat Diagn 2006;26:725-729.

16 Midro AT1, Panasiuk B, Tümer Z, Stankiewicz P, Silahtaroglu A, Lupski JR, Zemanova Z, Stasiewicz-Jarocka B, Hubert E, Tarasów E, Famulski W, Zadrozna-Tołwińska B, Wasilewska E, Kirchhoff M, Kalscheuer V, Michalova K, Tommerup N: Interstitial deletion 9q22.32-q33.2 associated with additional familial translocation $\mathrm{t}(9 ; 17)(\mathrm{q} 34.11 ; \mathrm{p} 11.2)$ in a patient with Gorlin-Goltz syndrome and features of Nail-Patella syndrome. Am J Med Genet A 2004;124A:179-191.

17 de Zwaan SE, Haass NK: Genetics of basal cell carcinoma. Australas J Dermatol 2010;51:81-92.

18 High A, Zedan W: Basal cell nevus syndrome. Curr Opin Oncol 2005;17:160-166.

19 Wang W, Wang J, Li J, Mao L, Guo F, Zhang B: New mutation of the patched homologue 1 gene in a Chinese family with naevoid basal cell carcinoma syndrome. Br J Oral Maxillofac Surg 2009;47:366-369.

20 Suzuki M, Hatsuse H, Nagao K, Takayama Y, Kameyama K, Kabasawa Y, Omura K, Yoshida M, Fujii K, Miyashita T: Selective haploinsufficiency of longer isoforms of PTCH1 protein can cause nevoid basal cell carcinoma syndrome. J Hum Genet 2012;57:422-426.

21 Kosaki R, Nagao K, Kameyama K, Suzuki M, Fujii K, Miyashita T: Heterozygous tandem duplication within the PTCH1 gene results in nevoid basal cell carcinoma syndrome. Am J Med Genet A 2012;158A:17241728.

22 Hirano T: At the heart of the chromosome: SMC proteins in action. Nat Rev Mol Cell Biol 2006;7:311-322.

-23 Remeseiro S, Cuadrado A, Losada A: Cohesin in development and disease. Development 2013;140:37153718.

-24 Wendt KS, Yoshida K, Itoh T, Bando M, Koch B, Schirghuber E, Tsutsumi S, Nagae G, Ishihara K, Mishiro T, Yahata K, Imamoto F, Aburatani H, Nakao M, Imamoto N, Maeshima K, Shirahige K, Peters JM: Cohesin mediates transcriptional insulation by CCCTC-binding factor. Nature 2008;451:796-801.

25 Santos JL, Carvalho E, Bezerra JA: Advances in biliary atresia: from patient care to research. Braz J Med Biol Res 2010;43:522-527.

-26 Vrouwe MG, Elghalbzouri-Maghrani E, Meijers M, Schouten P, Godthelp BC, Bhuiyan ZA, Redeker EJ, Mannens MM, Mullenders LH, Pastink A, Darroudi F: Increased DNA damage sensitivity of Cornelia de Lange syndrome cells: evidence for impaired recombinational repair. Hum Mol Genet 2007;16:1478-1487.

27 Heidinger-Pauli JM, Mert O, Davenport C, Guacci V, Koshland D: Systematic Reduction of Cohesin Differentially Affects Chromosome Segregation, Condensation, and DNA Repair. Curr Biol 2010;20:957963.

28 Revenkova E, Focarelli ML, Susani L, Paulis M, Bassi MT, Mannini L, Frattini A, Delia D, Krantz I, Vezzoni P, Jessberger R, Musio A: Cornelia de Lange syndrome mutations in SMC1A or SMC3 affect binding to DNA. Hum Mol Genet 2009;18:418-427.

29 Schär P, Fäsi M, Jessberger R: SMC1 coordinates DNA double-strand break repair pathways. Nucleic Acids Res 2004;32:3921-3929. 


\section{Cellular Physiology Cell Physiol Biochem 2015;35:270-280 and Biochemistry \\ Cao et al.: 9q31.1-q32 Deletion Patients and CdLS Have Common Features}

30 Akai Y, Kurokawa Y, Nakazawa N, Tonami-Murakami Y, Suzuki Y, Yoshimura SH, Iwasaki H, Shiroiwa Y, Nakamura T, Shibata E, Yanagida M: Opposing role of condensin hinge against replication protein A in mitosis and interphase through promoting DNA annealing. Open Biol DOI:10.1098/rsob.110023.

-31 Hirota T, Gerlich D, Koch B, Ellenberg J, Peters JM: Distinct functions of condensin I and II in mitotic chromosome assembly. J Cell Sci 2004;117:6435-6445.

-32 Hagstrom KA, Meyer BJ: Condensin and cohesin: more than chromosome compactor and glue. Nat Rev Genet 2003;4:520-534.

-33 Losada A, Hirano T: Dynamic molecular linkers of the genome: the first decade of SMC proteins. Genes Dev 2005;19:1269-1287.

34 Huang K, Jia J, Wu C, Yao M, Li M, Jin J, Jiang C, Cai Y, Pei D, Pan G, Yao H: Ribosomal RNA Gene Transcription Mediated by the Master Genome Regulator Protein CCCTC-binding Factor (CTCF) Is Negatively Regulated by the Condensin Complex. J Biol Chem 2013;288:26067-26077.

-35 Ebermann I, Scholl HP, Charbel Issa P, Becirovic E, Lamprecht J, Jurklies B, Millán JM, Aller E, Mitter D, Bolz $\mathrm{H}$ : A novel gene for Usher syndrome type 2: mutations in the long isoform of whirlin are associated with retinitis pigmentosa and sensorineural hearing loss. Hum Genet 2007;121:203-211.

- 36 Bedeschi MF1, Colombo L, Mari F, Hofmann K, Rauch A, Gentilin B, Renieri A, Clerici D: Unmasking of a Recessive SCARF2 Mutation by a 22q11.12 de novo Deletion in a Patient with Van den Ende-Gupta Syndrome. Mol Syndromol 2010;1:239-245. 